\title{
MESA VIBRATORIA ACCIONADA POR MÚSCULOS NEUMÁTICOS TELEOPERADA PARA EXPERIMENTACIÓN ON- LINE
}

\author{
Francisco Jesús Velasco González \\ E. T. S. de Nautica, c $\backslash$ Gamazo 1, 39004, Santander, velascof@unican.es
}

Luis M. Vega, Elías Revestido Herrero, Francisco J. Lastra, Sergio García, Ernesto Madariaga

E. T. S. de Nautica, c Gamazo 1, 39004, Santander, vegalm@unican.es, revestidoe@unican.es,

fjlastra@teisa.unican.es, sergio.garcia@unican.es, ernesto.madariaga@unican.es

\begin{abstract}
Resumen
El presente trabajo tiene como objetivo integrar el estudio de la dinámica estructural y las técnicas de control en el ámbito de la ingeniería, mediante el diseño y aplicación de un mesa vibratoria teleoperada para experimentación on-line. Para ello, se han propuesto una serie de experiencias docentes con la mesa, que han sido valoradas positivamente por los estudiantes.
\end{abstract}

Palabras clave: Músculo neumático, control, actuadores no lineales, vibraciones, dinámica estructural.

\section{INTRODUCCIÓN}

En el ámbito docente, una de las posibles aplicaciones que pueden tener las mesas de vibraciones es la de integrar el estudio de la dinámica estructural y las técnicas de control en ingeniería. Para ello, se diseña una mesa de pequeño tamaño, accionada por medio de músculos neumáticos y válvulas neumáticas proporcionales, que permiten un accionamiento flexible y fácilmente controlable. Por otra parte, dada la no linealidad del sistema de accionamiento, es posible introducir a los alumnos en el estudio de sistemas no lineales y su comportamiento.

Es posible diseñar distintos tipos de experiencias docentes con la mesa de vibraciones para para ilustrar al estudiante en diversos conceptos de dinámica estructural y demostrar conceptos de control estructural pasivos, pudiendo ampliarse su utilidad, posteriormente, a otros aspectos de dinámica estructural o al control activo de vibraciones.

Por otro lado, el convenio STCW (International Convention on Standards of Training Certification and Watchkeeping for Seafaders) de la IMO (International Maritime Organization) contiene la legislación marítima internacional que establece los estándares de capacitación profesional de las gentes de mar [1], Las enmiendas de Manila de 2010 al código de formación STCW, que terminó de implementarse en el año 2017, fomentan orientaciones interesantes para los centros de educación y formación marítima (MET), en concreto uno de los puntos del Convenio establece la necesidad de potenciar la educación a distancia y elearning, así como el uso de simuladores en la formación y en la actualización de conocimientos, teniendo en cuenta que el entorno de trabajo marítimo dificulta la actualización de conocimientos mediante la educación presencial tradicional. Con ello crea la base para que las administraciones marítimas nacionales permitan la formación de marinos por estas vías de acuerdo con las guías establecidas en la sección A-I/6 del Convenio, y poder emitir los correspondientes certificados de competencia [1] [2].

Hasta el momento, la única formación en que era obligatorio el uso de simuladores de acuerdo al convenio STCW ha sido el relativo al uso de Automatic Radar Plotting Aids (ARPA), a partir de las enmiendas de 2010 donde se establecen también como únicos métodos aceptados en la demostración de competencias profesionales los simuladores de cartas electrónicas y sistemas de información (ECDIS). Para todos los demás casos la formación mediante simuladores no se establece como obligatoria pero si como recomendable. Esta categoría de formación opcional con simuladores cubre la navegación, manejo del buque y de la carga, comunicación GMDSS y maquinaria de propulsión y auxiliar, pero aunque se impulsan las tecnologías utilizables en la instrucción no se definen modelos o guías específicas para la formación práctica basada en web.

En otro orden de cosas, la utilización de músculos neumáticos presenta múltiples ventajas como la carencia de mantenimiento, la flexibilidad de utilización, la elevada relación potencia/peso comparada con los cilindros neumáticos [3], su resistencia a ambientes extremos, el desarrollo de 
fuerzas de hasta 4000N [4], y un periodo de vida de al menos 10 millones de ciclos [5]. La relación potencia peso depende del tipo de músculo y varía entre 1 y 10 $\mathrm{kW} / \mathrm{kg}[6]$.

Es ampliamente conocido, que un músculo neumático solo puede proporcionar una fuerza de tracción y no puede transmitir fuerzas en compresión, esta fuerza de tracción tiene un máximo al comienzo de la contracción y cae a cero cuando la carrera es nula [5]. Posee una dependencia no lineal entre la fuerza desarrollada, su contracción y la presión interna, lo que junto a los efectos de histéresis representa su principal desventaja al ser difícil el posicionamiento preciso y su control [7]. Su comportamiento no lineal es una de las razones de que su uso no esté muy extendido [8], aunque actualmente están siendo integrados en sistemas robotizados especialmente gracias a su ligereza y su potencia [9].

Los músculos neumáticos tienen su propio comportamiento dinámico, como histéresis [10], la característica nolineal del caudal de las servoválvulas y del propio actuador [11], efectos termodinámicos del aire [12]. Actualmente no hay un estado del arte totalmente desarrollado debido a su importante característica de nolinealidad [13]. La mayor parte de los modelos actuales estudian las características de la fuerza estática, y lo interpretan como una combinación de un sistema resorte-amortiguador [14].

En este trabajo se propone la aplicación de un sistema de experimentación teleoperado con mesa de vibraciones accionada por musculo neumático, que permite implicar al alumno en el estudio interdisciplinar de sistemas no lineales neumáticos, y respuesta de sistemas mecánicos, de tal forma que el estudiante pueda participar en su desarrollo. Además, se incluye un soporte software para la experimentación on line del estudiante satisfaciendo los requisitos de educación a distancia del convenio STCW anteriormente citados.

\section{MODELO MATEMÁTICO}

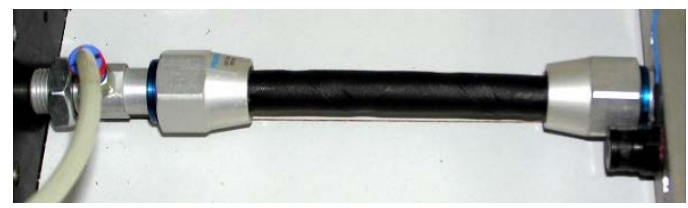

Figura 1 Músculo neumático FESTO

La dinámica del músculo neumático (figura 1), utilizado en este trabajo, está supedita a la dinámica del fluido del aire en su interior y por el caudal de aire que caracteriza el control del músculo, siendo uno de los causantes de su no linealidad su dependencia de los efectos termodinámicos. Un punto inicial es relacionar la presión en el interior del músculo con el flujo de aire de alimentación $\left(\dot{m}_{l}\right)$, para ello se puede partir de la ecuación de los gases perfectos ya que describe la dependencia con la masa de gas:

$$
m=\frac{p V}{R T}
$$

Dónde: m es la masa de aire en el interior del músculo, $\mathrm{p}$ es la presión en el interior del músculo, $\mathrm{V}$ es el volumen interno del músculo, $\mathrm{R}$ es la constante del gas y $\mathrm{T}$ es la temperatura del gas.

Como el tubo del músculo consiste de un elastómero, solo pasa el calor a través del material parcialmente, y asumiendo que la presión de suministro de aire permanece constante, puede suponerse que cualquier variación del volumen o la presión del músculo se comportará entre la condición ideal isoterma y la adiabática y podrá ser descrita como una ley politrópica [15]:

$$
p_{1} V_{1}^{\gamma}=p_{2} V_{2}^{\gamma}
$$

Donde $\mathrm{p}_{\mathrm{i}}$ es la presión en el interior del músculo, siendo 1 y 2 dos estados de presión distintos, $\mathrm{V}_{\mathrm{i}}$ es el volumen de la cámara interior del músculo, y el exponente politrópico $\checkmark$ se ha valorado en algunos trabajos como $\gamma=1,26$. [5].

Combinando (1) y (2) se llega a una expresión para la variación de presión [16]:

$$
\frac{d p}{d t}=\frac{\gamma}{V_{(s)}}\left[r T q_{m}(u, p)-p \frac{d V}{d s} \dot{s}\right]
$$

Donde s es la posición del extremo (se corresponde con la posición que tendría el del pistón en el caso de comportarse como un cilindro), $\mathrm{q}_{\mathrm{m}}(\mathrm{u}, \mathrm{p})$ representa el flujo másico $\left(\frac{d m}{d t}=q_{m}(u, p)\right)$ y u representa la entrada de tensión a la válvula proporcional,

A este efecto habría que añadir el propio comportamiento dinámico del músculo, como es su histéresis [10] y la característica no lineal del caudal de las servoválvulas y del propio actuador [11].

El primer modelo para describir el músculo fue introducido McKibben [17], y se basaba en la energía necesaria para cambiar el volumen de aire interno, despreciando los efectos de la elasticidad de la membrana.

$$
F_{\text {McKibben }}=-p \frac{d V}{d L}=p \frac{L_{\text {Fibra }}^{2}\left(3 \cos ^{2} \theta-1\right)}{4 \pi n^{2}}
$$

Esta expresión no describe el funcionamiento de los músculos MAS de Festo, puesto que estos almacenan energía en la membrana deformada y el McKibben no lo hace. Una corrección posterior $[12,18]$ corregía este efecto añadiendo dos factores adicionales, y posteriormente se presentó un modelo más preciso por Sarosi et al [19], en el se considera que la fuerza de contracción, o de elongación, producida por un músculo neumático depende de su geometría y de las propiedades de los parámetros materiales de las capas interior y exterior del tubo elástico, así como de la presión del aire “p” [20], para ello se introdujo el parámetro de contracción k:

$$
k=\frac{l_{0}-l}{l}
$$


Siendo $l_{0}$ la longitud inicial del músculo en reposos y 1 la longitud el músculo presurizado.

Sarosi et al [19] incluyeron algoritmos con 6 parámetros desconocidos para la fuerza generada por los músculos neumáticos, obteniendo una muy alta precisión con músculos neumáticos de $20 \mathrm{~mm}$ de diámetro interior [21].

$$
F_{\text {Sarosi }}=\left(a_{1} p+a_{2}\right) e^{a_{3} k}+a_{4} p k+a_{5} p+a_{6}
$$

Donde $\mathrm{a}_{1}, \mathrm{a}_{2}, \mathrm{a}_{3}, \mathrm{a}_{4} \mathrm{a}_{5}, \mathrm{a}_{6}$ con constantes desconocidas, que puede obtenerse por regresión no lineal a partir de los datos experimentales.

Las fuerzas desarrolladas y los volúmenes del músculo también se han aproximado por funciones polinomiales [15]:

$$
\begin{aligned}
& F\left(k_{j}, p_{j}\right)=\left(\sum_{i=0}^{3} a_{i} k_{j}^{i}\right) p_{j}+\sum_{i=0}^{3} b_{i} k_{j}^{i}+b_{4} k_{j}^{\frac{b_{5}}{2}} \\
& V\left(k_{j}\right)=\sum_{i=0}^{3} c_{i} k_{j}^{i}
\end{aligned}
$$

Con $a_{i}, b_{i}, b_{4} b_{5} c_{i} \in \mathbb{R}$. Los parámetros pueden obtenerse también mediante regresión lineal con datos experimentales.

En [5] se presentó un modelo específico para el músculo festo. Este modelo es una combinación de presión y área virtual de la sección y una fuerza dependiente de la longitud. La idea es que el músculo se comporta como una combinación de pistón neumático con un área de pistón variable y un resorte mecánico que contrarresta la expansión del músculo.

$$
\begin{array}{r}
F_{\text {Hildebrandt }}=p A(L)-F_{c}(L)=p \sum_{j=0}^{2} c_{j} l^{J}- \\
\left(\sum_{J=0}^{3} d_{j} L^{j}+d_{4} L^{2 / 3}\right)
\end{array}
$$

Por otra parte, según Sarosi et al [19], el músculo neumático se puede considerar como un sistema de un grado de libertad (figura 3) con amortiguamiento no lineal y fuerza de contracción F. En este caso las vibraciones del sistema masa-resorte con amortiguamiento se deben a una fuerza no lineal. La ecuación general del sistema es:

$$
m \ddot{x}=-F_{\left(k_{(x)}\right)}-c_{\left(k_{(x)}\right)} \cdot \dot{x}+m g
$$

Donde $\mathrm{m}$ es la masa del sistema, $\mathrm{x}$ es la elongación, $\mathrm{g}$ es la constante de gravedad y $\mathrm{F}$ es la fuerza no lineal a presión constante (ecuaciones 4 a 9).

El coeficiente de rigidez $\mathrm{k}$ y el coeficiente de amortiguamiento c también con funciones no lineales del desplazamiento $\mathrm{x}$.

Como ejemplo para la expresión obtenida por Sarosi et al [19] la rigidez del modelo en el caso de presión constante se calcula como:

$$
\begin{array}{r}
k=k_{(k)}=\frac{d F(l)}{d l}=\frac{d F(k)}{l_{0} d k}=\frac{1}{l_{0}} \frac{d F(k)}{d k}=\frac{1}{l_{0}} \frac{d F(p, k)}{d k}= \\
\frac{1}{l_{0}} \frac{d\left(\left(a_{1} p+a_{2}\right) e^{\left.a_{3} k_{+} a_{4} p k+a_{5} p+a_{6}\right)}\right.}{d k}= \\
\frac{\left(a_{1} p+a_{2}\right) a_{3} e^{a_{3} k_{+} a_{4} p}}{l_{0}}
\end{array}
$$

y el coeficiente de amortiguamiento $\mathrm{c}(\mathrm{x})$ se obtiene de acuerdo con la curva de histéresis de la curva de fuerza-contracción [22]. La histéresis se produce debido a la fricción entre el tubo elástico y sus refuerzos [19].

$$
c=2 \xi \sqrt{\frac{m}{l_{0}}\left[\left(a_{1} p+a_{2}\right) a_{3} e^{a_{3} k}+a_{4} p\right]}
$$

Los sistemas mecánicos unidos a la masa desplazada por los actuadores, en este caso los músculos neumáticos, responden a las perturbaciones del movimiento generado según sus frecuencias naturales.

En este trabajo se estudian sistemas de un grado y dos grados de libertad. Como ejemplo de un grado de libertad, se tiene una masa $\mathrm{M}$ unida a la base por pilares rígidos, ver figura 2. En esta figura, la sección recta de los pilares tiene momentos de inercia Ix e Iy, el material tiene módulo de elasticidad E, la altura del cuerpo es h, y la placa tiene masa $\mathrm{M}$ mucho mayor que la de los pilares por lo que se considera la masa de estos últimos despreciable.

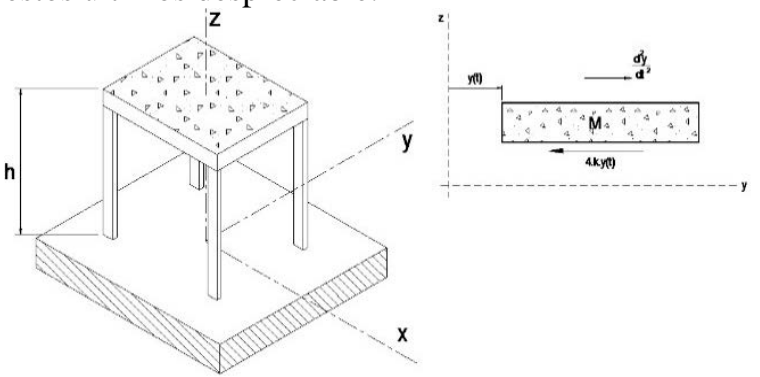

Figura 2 Modelo de estudio de un grado de libertad

La ecuación del movimiento en la dirección y es (figura 2):

$$
\begin{aligned}
& \sum F_{y}=-4 k y=M \ddot{y} \\
& \ddot{y}+\frac{48 E I_{x}}{M h^{3}} y=0
\end{aligned}
$$

Y la frecuencia propia de la oscilación libre:

$$
\omega=\sqrt{\frac{48 E I_{x}}{M h^{3}}}
$$

Un ejemplo de sistemas con varios grados de libertad se muestra en la figura 3 .

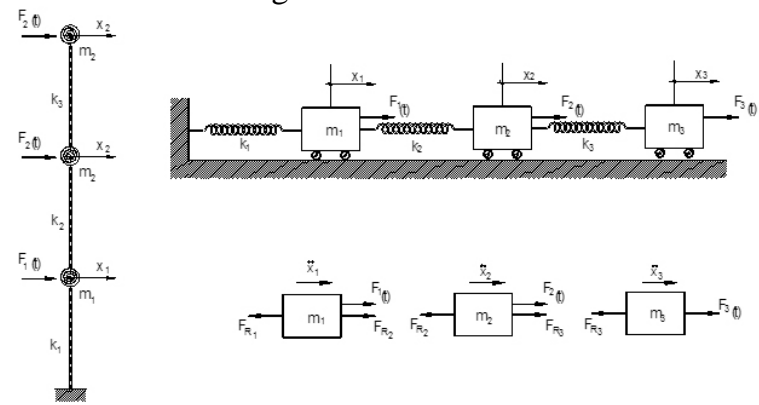

Figura 3 Modelo de varios grados de libertad 
Donde las fuerzas externas aplicadas se suman a las fuerzas de resorte equivalente Fr y las fuerzas de inercia Fi, obteniéndose [23]:

$$
\begin{gathered}
{\left[\begin{array}{ccc}
m_{1} & 0 & 0 \\
0 & m_{2} & 0 \\
0 & 0 & m_{3}
\end{array}\right]\left\{\begin{array}{l}
\ddot{x}_{1} \\
\ddot{x_{2}} \\
\ddot{x_{3}}
\end{array}\right\}+} \\
{\left[\begin{array}{ccc}
k_{1}+k_{2} & -k_{2} & 0 \\
-k_{2} & k_{2}+k_{3} & -k_{3} \\
0 & -k_{3} & k_{3}
\end{array}\right]\left\{\begin{array}{l}
x_{1} \\
x_{2} \\
x_{3}
\end{array}\right\}=\left\{\begin{array}{l}
0 \\
0 \\
0
\end{array}\right\}} \\
{[M]\{\ddot{x}\}+[K]\{x\}=0}
\end{gathered}
$$

Donde $\mathrm{m}_{\mathrm{i}}, \mathrm{k}_{\mathrm{i}}, \mathrm{x}_{\mathrm{i}} \mathrm{y} \ddot{x}_{\mathrm{l}}$ son la masa, la rigidez, el desplazamiento y la aceleración de cada masa, y [M] y [K] son las matrices de masa y de rigidez respectivamente.

La solución de esta ecuación para una estructura de varios grados de libertad se puede suponer de la forma:

$$
\{A\} \cos (\omega t)+\{A\} \operatorname{sen}(\omega t)=\{x\} \operatorname{sen}(\omega t+\alpha)
$$

Sustituyendo en la ecuación del movimiento:

$$
\left(-\omega^{2}[M]+[K]\right)\{x\} \operatorname{sen}(\omega t+\alpha)=0
$$

Las formas modales son patrones de desplazamiento de la estructura, es decir la posición relativa de las distintas masas concentradas de la estructura cuando se excita a una frecuencia igual a una de sus frecuencias naturales, tanto las formas modales como los grados de libertad de cada forma modal se asocian con una frecuencia natural, así que el cálculo de las formas modales se puede desarrollar después de determinar las frecuencias naturales.

De las ecuaciones anteriores:

$$
\left(-\omega^{2}[M]+[K]\right)\{x\}=0
$$

Sustituyendo el valor de cada frecuencia en esta ecuación, solo queda como valor desconocido el vector $\{x\}$ de desplazamiento de las masas. Las ecuaciones obtenidas al sustituir los valores de las frecuencias naturales en la ecuación anterior son dependientes lo que hace imposible resolver el sistema de ecuaciones para las amplitudes de desplazamiento, pero como solo se necesita conocer las relaciones entre las amplitudes se puede suponer cualquier valor para una de las variables y las ecuaciones adicionales se resuelven en función de ese parámetro elegido.

\section{DESCRIPCIÓN DE LA MESA VIBRATORIA}

El diseño básico de la mesa vibratoria desarrollado en este trabajo se mostrado en la figura 4. Esta configuración, desplaza una carga (m=masa) por medio de dos músculos neumáticos en una guía lineal. El actuador de músculo neumático utilizado es de la marca Festo, y se han utilizado como actuador una válvula proporcional por músculo ya que es la implementación más extendida en la literatura técnica [7]. Además, la mesa se ha instrumentado con acelerómetros piezoeléctricos y capacitivos para la medición de movimientos, ver figura 5.

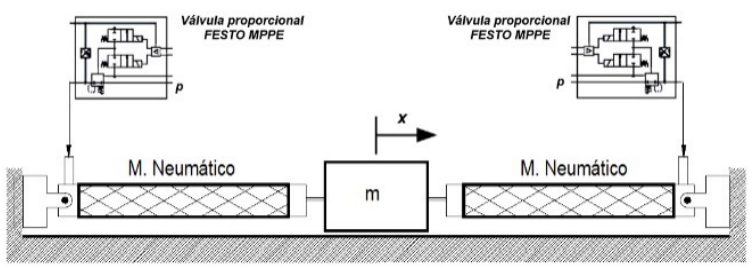

Figura 4 Modelo de mesa vibratoria de un eje

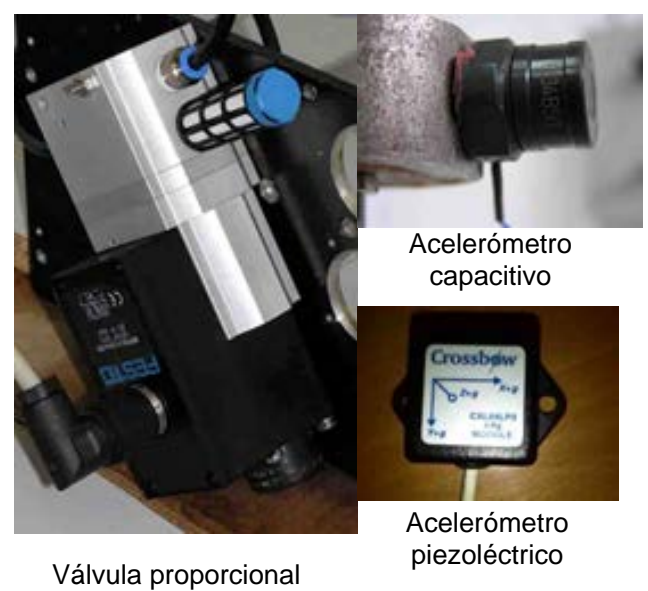

Figura 5 Elementos de la mesa vibratoria

Se ha desarrollado un soporte software para la adquisición de datos de los acelerometros y control de la mesa vibratoria mediante tarjeta de aquisición de datos, ver figura 6.

Asimismo, gracias al desarrollo de una página web donde se ha incrustado la aplicación de adquisición de datos de la mesa vibratoria es posible el acceso a distancia del estudiante a los datos que se están midiendo mediante un pc que disponga de una conexión a internet. Esta página, está basa en servidor web donde se publican los datos del software desarrollados para la mesa vibratoria.

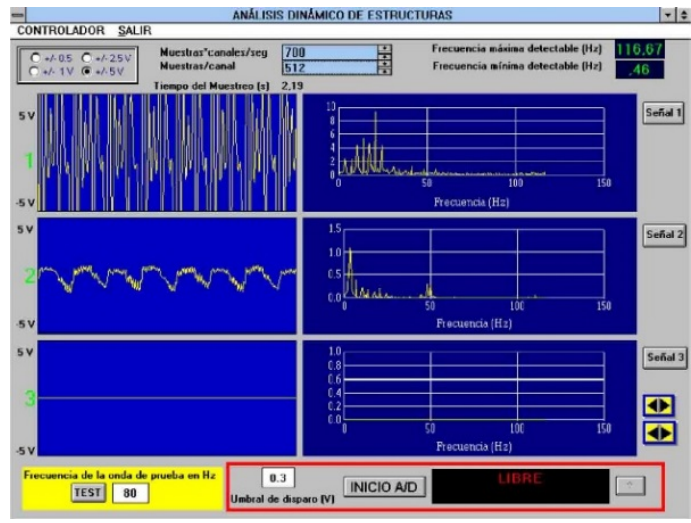

Figura 6 Soporte software de la mesa vibratoria

\section{EXPERIENCIAS DOCENTES}




\subsection{PRESTACIONES DOCENTES DE LA MESA VIBRATORIA}

Las experiencias docentes con la mesa vibratoria se plantea como un proyecto interactivo profesorestudiante de tecnologías navales, donde se ilustra y consolidan conceptos de ingeniería de automatización y control, ingeniería estructural y mecánica, instrumentación de control, actuadores, adquisición de datos y su tratamiento; actuando por lo tanto como un instrumento integrador de carácter multidisciplinar de estas materias.

Previo a la utilización de la mesa, el profesor deberá instruir al estudiante en aspectos básicos de la neumática proporcional. Después, bajo la supervisión del profesor el estudiante puede experimentar con la mesa, de forma autónoma o colaborativa, para la obtención de las distintas respuestas de sistemas mecánicos.

La primera experimentación realizada hace referencia a la caracterización del músculo neumático, utilizando una célula de carga (figura 7) se anotan los valores de las fuerzas desarrolladas por el músculo para distintas presiones y porcentajes de retracción o acortamiento del músculo, con los datos obtenidos el alumno caracteriza su comportamiento mediante técnicas de regresión no lineal ajustando las medidas a los distintos modelos de las ecuaciones 6 a 12, de forma que interpreten el carácter no lineal del sistema.

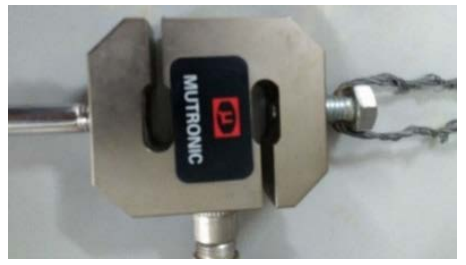

Figura 7 Célula de carga

La experimentación permite aprender a utilizar sensores industriales como son los acelerómetros con sus acondicionadores de señal, así como descubrir la utilidad de dispositivos de uso común para el análisis de estructuras con fines académicos.

Los alumnos experimentan con la respuesta de la mesa y del sistema mecánico en ella instalado, mediante las señales que envían a la válvula proporcional, mientras que en el software desarrollado se registran los datos de los acelerómetros y se obtiene la FFT de dicha señal; los datos de la señal de movimiento se presentan gráficamente en función del tiempo con el correspondiente espectro en frecuencia, caracterizando el sistema estudiado y sirviendo como herramienta de discusión por parte de los alumnos.

De igual manera, los alumnos comprueban la respuesta de los sistemas mecánicos estudiados, de uno y dos grados de libertad, cuya frecuencia teórica es conocida por medio de cálculos sencillos, instalan dichos sistemas mecánicos en la mesa y la someten a distintos movimientos forzados con frecuencias cercanas a la frecuencia natural de los sistemas estudiados.De este modo el estudiante estudia el sistema teórico y experimenta con el modelo real, estudiando su respuesta en frecuencia.

A modo de ejemplo se han realizado experiencias con los alumnos con la mesa soportando un poste empotrado con una masa concentrada y con dos masas concentradas (Figura 8).

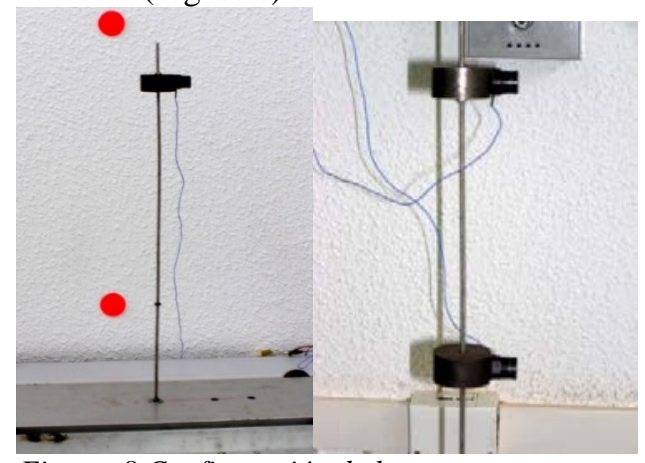

Figura 8 Configuración de la mesa con un poste empotrado en su base y una masa concentrada (izquierda) $y$ dos masas concentradas (derecha).

\section{RESULTADOS}

Resulta difícil cuantificar la mejora en el rendimiento académico de los alumnos, puesto que las asignaturas están formadas por muchos más contenidos que los tratados en esta experiencia, siendo esta actividad una pequeña parte de las actividades totales.

$\mathrm{Si}$ es posible valorar cualitativamente la mayor implicación de los alumnos al plantarles una actividad interactiva, con aplicaciones prácticas en su futura profesión, la cual es positiva al aumentar la participación activa del alumnado.

La experiencia docente previa advierte de una percepción abstracta de conceptos de sistemas mecánicos y de control por parte de los alumnos, lo que en ocasiones dificulta su aprendizaje a una parte de los mismos; el trabajo propuesto en este artículo ha permitido detectar una mayor implicación del alumnado, que minimiza parte de esa percepción abstracta acercándola a una más cercana a la real, y mejorando su implicación.

A través de la experimentación y la discusión en común por parte de los alumnos, deducen aspectos como el movimiento no sinusoidal del músculo neumático y por lo tanto de la mesa accionada, y con ello la utilidad de la FFT para obtener las frecuencias del primer modo y los armónicos correspondientes, de este modo los alumnos comprenden y valorar la importancia de calcular la respuesta en frecuencia de un sistema mecánico.

Se muestra el caso estudiado con los alumnos de dos masas concentradas en un poste empotrado, (Figura 9) una en su extremo y otra en la posición media. 
El primer paso que han de realizar los alumnos es la determinación teórica de las frecuencias naturales y los modos normales de vibración, lo que realizaron mediante la determinación previa de los coeficientes de influencia mediante la integral de Mohr y el método de multiplicación de diagramas de Vereshchaguin (figura 10).

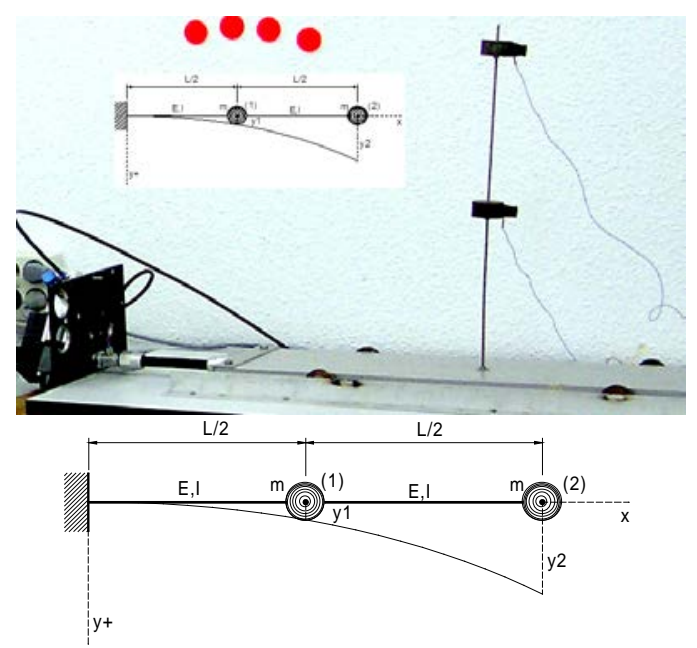

Figura 9 Sistema poste empotrado con dos masas

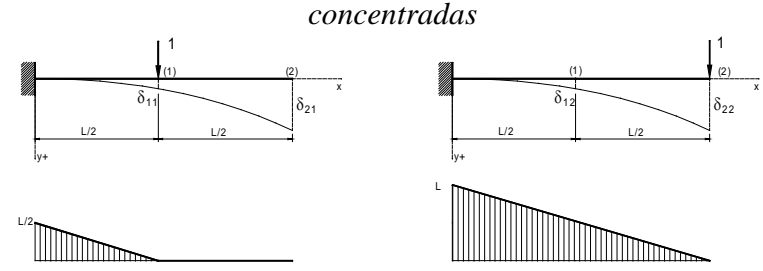

Figura 10 Coeficientes de influencia

$$
\begin{gathered}
\delta_{11}=\frac{L^{3}}{24 E I} \\
\delta_{22}=\frac{L^{3}}{3 E I} \\
\delta_{12}=\delta_{21}=\frac{L^{3}}{48 E I}
\end{gathered}
$$

Y deduciendo los desplazamientos y1 e y2 por superposición de efectos:

$$
\begin{aligned}
& y_{1}=\delta_{11} P_{1}+\delta_{12} P_{2} \\
& y_{2}=\delta_{21} P_{1}+\delta_{22} P_{2}
\end{aligned}
$$

Considerando que las fuerzas de gravedad se equilibran con la reacción vertical en el empotramiento, las únicas fuerzas que aparecen sobre la columna son las fuerzas de inercia de D’Alembert y las reacciones dinámicas en los apoyos (las que surgen por efecto del movimiento). Ahora se pueden escribir las ecuaciones de los desplazamientos de las masas:

$$
\begin{aligned}
& y_{1}=-\delta_{11} m_{1} \frac{d^{2} y_{1}}{d t^{2}}-\delta_{12} m_{2} \frac{d^{2} y_{2}}{d t^{2}} \\
& y_{2}=-\delta_{21} m_{1} \frac{d^{2} y_{1}}{d t^{2}}-\delta_{22} m_{2} \frac{d^{2} y_{2}}{d t^{2}}
\end{aligned}
$$

Que con solución general de la forma:

$$
\begin{aligned}
& y_{1}=A_{1} \operatorname{sen}(\omega t+\alpha) \\
& y_{2}=A_{2} \operatorname{sen}(\omega t+\alpha)
\end{aligned}
$$

Permite obtener la ecuación de frecuencias naturales:

$\left.\begin{array}{l}\omega_{1}^{2} \\ \omega_{2}^{2}\end{array}\right\}$

$$
=\frac{\delta_{11} m_{1+} \delta_{11} m_{1} \pm \sqrt{\left(\delta_{11} m_{1}-\delta_{22} m_{2}\right)^{2}+4 m_{1} m_{2} \delta_{12}^{2}}}{2 m_{1} m_{2}\left(\delta_{11} \delta_{22}-\delta_{12}^{2}\right)}
$$

Con las dos masas concentradas de igual valor $\mathrm{m}_{1}=\mathrm{m}_{2}=\mathrm{m}$, se obtienen las frecuencias naturales:

$$
\begin{aligned}
\omega_{1} & =10,99 \sqrt{\frac{E I}{m L^{3}}} \mathrm{rad} / \mathrm{s} \\
\omega_{1} & =1,65 \sqrt{\frac{E I}{m L^{3}}} \mathrm{rad} / \mathrm{s}
\end{aligned}
$$

Y las razones modales:

$$
\begin{aligned}
& \left(\frac{A_{1}}{A_{2}}\right)_{\omega_{1}}=\frac{1-\delta_{11} m_{1} \omega_{1}^{2}}{\delta_{12} m_{2} \omega_{1}^{2}}=\frac{\delta_{12} m_{1} \omega_{1}^{2}}{1-\delta_{22} m_{2} \omega_{1}^{2}} \\
& \left(\frac{A_{2}}{A_{1}}\right)_{\omega_{2}}=\frac{1-\delta_{11} m_{1} \omega_{2}^{2}}{\delta_{12} m_{2} \omega_{2}^{2}}=\frac{\delta_{12} m_{1} \omega_{2}^{2}}{1-\delta_{22} m_{2} \omega_{2}^{2}}
\end{aligned}
$$

Que para los datos del ejemplo estudiado resultan unos valores teóricos de:

$$
\begin{aligned}
& \left(\frac{A_{1}}{A_{2}}\right)_{\omega_{1}}=3,12 \\
& \left(\frac{A_{2}}{A_{1}}\right)_{\omega_{2}}=-0,32
\end{aligned}
$$

Siendo los modos normales los mostrados en la figura 11
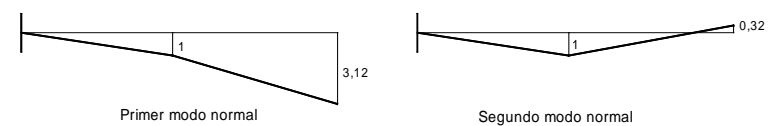

Figura 11Modos normales de vibración teóricos

Realizada la experimentación y sometiendo la mesa a perturbaciones con valores de frecuencia correspondientes al primer modo de vibración, se obtienen medidas correspondientes de los desplazamiento que siguen el comportamiento deducido en el desarrollo teórico (Figura 12)

Lo que se corresponde con el comportamiento teórico, de este modo por medio de la experimentación autónoma, analizando los datos obtenidos, el estudiante comprende el concepto de respuesta de un sistema dinámico de un sistema mecánico, así como los efectos de cambio de características físicas (como su rigidez) en las frecuencias de resonancia.

Del mismo modo en al figura 13 se muestra un ejemplo de los resultados obtenidos por los alumnos, sometiendo a la estructura estudiada a una perturbación cuya frecuencia es cercana al segundo modo normal del vibración. Donde se observan los dos picos de frecuencia en la imagen de la FFT, siendo el 
mayor de ellos, el correspondientes a la frecuencia del segundo modo y el deplazamiento relativo de las dos masas, con un comipotratmiento muy cercano al esperado por su desarrollo teórico.
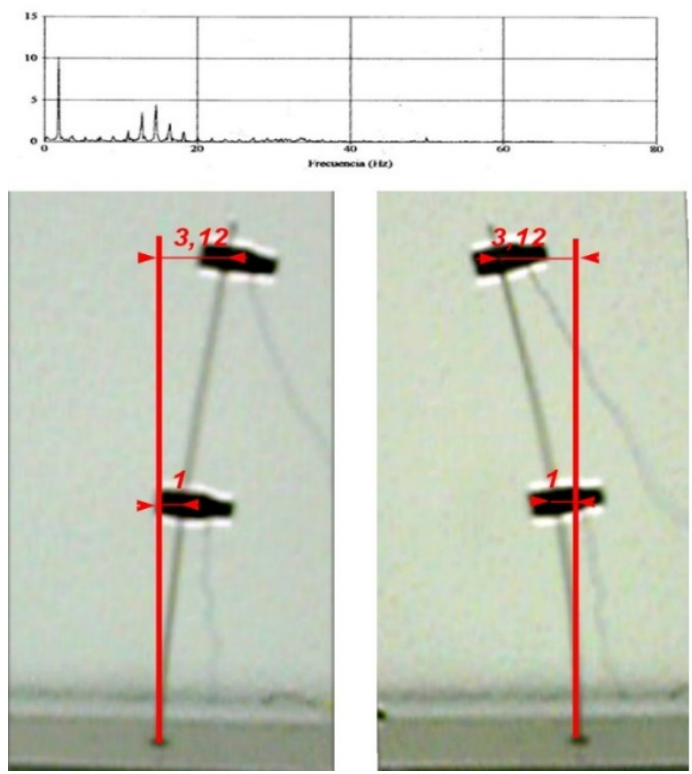

Figura 12 primer modo de vibración.

Debido a las limitaciones de funcionamiento del músculo neumático las frecuencias útiles de trabajo se encuentran entre 2 y $10 \mathrm{~Hz}$ aproximadamente, lo que permite al estudiante experimentar también con los acelerómetros MEMS presentes en los teléfonos móviles, que suelen tener una frecuencia máxima de medida de $100 \mathrm{~Hz}$, y aunque su precisión es escasa, es suficiente para ser utilizado en una aplicación docente, y su uso estimula la atención y la participación del alumno, por no ser habitual como equipo docente.

\section{CONCLUSIONES}

Se observa una mayor implicación del estudiante en la materia dada la utilidad práctica de las experiencias docentes diseñadas. Además, permite la participación del estudiante en su desarrollo completo desde el estudio de los elementos individuales, a su programación, interconexión y aplicación práctica, finalizando con el proceso de análisis de los datos. De este modo el alumno valora la aplicación práctica por medio de métodos matemáticos como el análisis por transformada rápida de Fourier FFT, y el significado de los resultados obtenidos.

Los alumnos valoran positivamente las actividades, y consideran que las tareas llevadas a cabo les permiten obtener una visión general de las aplicaciones de la automática e instrumentación sobre equipos mecánicos.

Se ha mejorado la adquisición de conocimientos, y su correcta interpretación en lo referente a sistemas de medida y tratamiento de las señales.

\section{Agradecimientos}

Este trabajo ha contado con el apoyo del MINECO y de la Universidad de Cantabria en una convocatoria de proyectos de innovación docente.
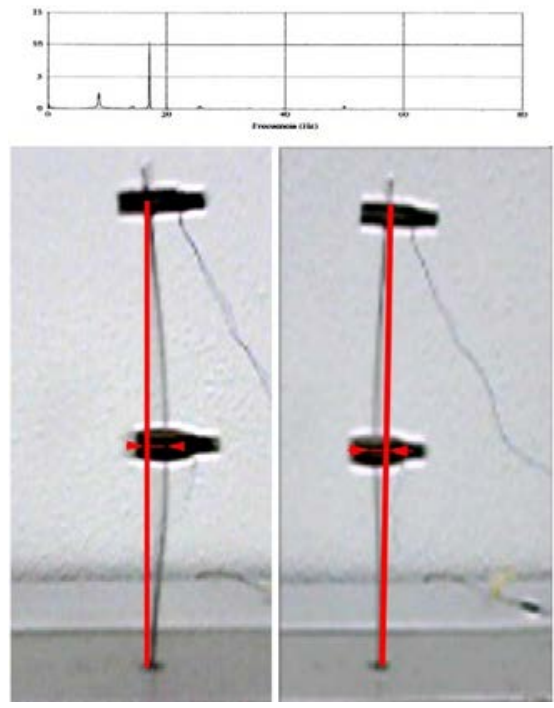

Figura 13 Comportamiento segundo modo normal de vibración.

\section{English summary}

\section{TELEOPERATED VIBRATING TABLE WITH PNEUMATIC MUSCLE FOR ON-LINE EXPERIMENTATION}

\begin{abstract}
The present work aims to integrate the study of structural dynamics and control techniques in the field of engineering, through the design and application of a teleoperated vibrating table for on-line experimentation. To this end, a series of teaching experiences with the table have been proposed, which have been positively evaluated by the students.
\end{abstract}

Keywords: Pneumatic muscle, control, non-linear actuators, vibrations, structural dynamics

\section{Referencias}

[1] IMO, «The Manila Amendments to the Seafarers' Training, Certification and Watchkeeping (STCW) Code» de Conference of parties to the international convention on standards of training, certification and watchkeeping for seafarers, Manila, 210.

[2] I. T. W. Federation, «STCW a guide for seafaders - taking into account the 2010 Manila amendments» 2010.

[3] F. Daerden, D. Lefeber, "Pneumatic artificial muscles: actuator for robotics and automation”, Eur. J. Mech. Environ. Eng. 47 (1) (2002) 11-21. 
[4] S. V. Krichel , O. Sawodny \& A. Hildebrandt "Tracking control of a pneumatic muscle actuator using one servovalve”. In American Control Conference (ACC), pp. 4385-4390, 2010.

[5] A. Hildebrandt, O. Sawodny, R. Neumann \& A. Hartmann. "Cascaded control concept of a robot with two degrees of freedom driven by four artificial pneumatic muscle actuators”. In American Control Conference, 2005. pp. 680685, 2005.

[6] J.H. Lilly, Adaptive tracking for pneumatic muscle actuators in bicep and tricep configurations, IEEE Trans. Neural Syst. Rehabil. Eng. 11 (3) (2003) 333-339.

[7] B. Tondu, S. Ippolito, J. Guiochet, A. Daidie, A Seven-degrees-of-freedom Robot-arm Driven by Pneumatic Artificial Muscles for Humanoid Robots, The International Journal of Robotics Research 24 (4) (2005) 257-274.

[8] F. Takemura, S.R. Pandian, S. Kawamura, Y. Hayakawa, "Observer design for control of pneumatic cylinder actuators," in "PTMC 99, Bath Workshop on Power Transmission and Motion Control”, Bath, Sep 8.-10.9.1999, pp. 223-236, 1999.

[9] Boblan, I.; Maschuw, J.; Engelhardt, D.; Schulz, A.; Schwenk, H.; Bannasch, R.; Rechenberg, I. A human-like robot hand and arm with fluidic muscles: Modelling of a muscle driven joint with an antagonistic setup. In Proceedings of the 3rd International Symposium on Adaptive Motion in Animals and Machines, Ilmenau, Germany, 2530 September 2005.

[10] Lin, C.J.; Lin, C.R.; Yu, S.K.; Chen, C.T. Hysteresis modeling and tracking control for a dual pneumatic artificial muscle system using Prandtl-Ishlinskii model. Mechatronics 2015, 28, 35-45.

[11] F. Takemura, S.R. Pandian, S. Kawamura, Y. Hayakawa, "Observer design for control of pneumatic cylinder actuators," in "PTMC 99, Bath Workshop on Power Transmission and Motion Control”, Bath, pp. 223-236, September, 1999.

[12] Andrikopoulos, G.; Nikolakopoulos, G.; Manesis, S. Incorporation of thermal expansion in static force modeling of pneumatic artificial muscles. In Proceedings of the 2015 23rd Mediterranean Conference on Control and Automation (MED), Torremolinos, Spain, 16-19 June 2015; pp. 414-420.

[13] G. Tao, P.V. Kokotovic," Adaptive control of systems with actuator and sensor nonlinearities", in John Wiley \& Sons, INC., New York, 1996.

[14] Kerscher, T.; Albiez, J.; Zollner, J.M.; Dillmann, R. Evaluation of the dynamic model of fluidic muscles using quick-release. In Proceedings of the First IEEE/RAS-EMBS International Conference on Biomedical Robotics and Biomechatronics, Pisa, Italy, 20-22 February 2006; pp. 637-642.

[15] A. Hildebrandt, O. Sawodny, and R. Neumann. A cascaded tracking control concept for pneumatic muscle actuators. In European Control Conference 2003 (ECC03), Cambridge UK, (CD-Rom), 2003.

[16] Chikh, L., Poignet, P., Pierrot, F., \& Michelin, M. (2010, June). A predictive robust cascade position-torque control strategy for Pneumatic Artificial Muscles. In American Control Conference (ACC), 2010 (pp. 6022-6029). IEEE.

[17] Boblan, I. Modellbildung und Regelung Eines Fluidischen Muskelpaares. Ph.D. Thesis, Technical University of Berlin, Berlin, Germany, 2010

[18] Andrikopoulos, G.; Nikolakopoulos, G.; Manesis, S. Novel Considerations on static force modeling of pneumatic muscle actuators. IEEE/ASME Trans. Mechatron. 2016, 21, 26472659.

[19] Sárosi, J.; Biro, I.; Nemeth, J.; Cveticanin, L. Dynamic modeling of a pneumatic muscle actuator with two-direction motion. Mech. Mach. Theory 2015, 85, 25-34.

[20] F., Daerden, Conception and realization of pleated artificial muscles and their use as compliant actuation elements. PhD Dissertation, Vrije Universiteit Brussel, Faculteit Toegepaste Wetenschappen Vakgroep Werktuigkunde, 1999, p. 5-33.

[21] Sarosi, J., \& Fabulya, Z. (2012). Mathematical analysis of the function approximation for the force generated by pneumatic artificial muscle. Transaction on Mechanics, Scientific Bulletin of the Politehnica, University of Timisoara, 57(71), 59-64:

[22] Wickramatunge, K.C.; Leephakpreeda, T. Study on mechanical behaviors of pneumatic artificial muscle. Int. J. Eng. Sci. 2010, 48, 188-198.

[23] Xu, Y., Hua, H., \& Han, J. (2008). Modeling and controller design of a shaking table in an active structural control system. Mechanical Systems and Signal Processing, 22(8), 1917-1923.

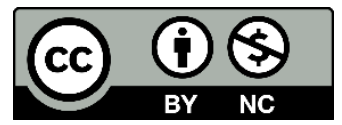
(C) 2018 by the authors. Submitted for possible open access publication under the terms and conditions of the Creative Commons Attribution CC-BY-NC $3.0 \quad$ license (https://creativecommons.org/licenses/by-nc/3.0). 